\title{
Maternal gestational weight gain and offspring's risk of cardiovascular disease and mortality
}

\author{
S Bhattacharya, ${ }^{1} \mathrm{G}$ McNeill, ${ }^{1,2}$ E A Raja, ${ }^{1}$ K Allan, ${ }^{1}$ H Clark, ${ }^{1}$ R M Reynolds, ${ }^{3}$ \\ J E Norman, ${ }^{3}$ P C Hannaford ${ }^{1}$
}

${ }^{1}$ Institute of Applied Health Sciences, University of Aberdeen, Aberdeen, UK ${ }^{2}$ Rowett Institute of Nutrition and Health, University of Aberdeen, Aberdeen, UK ${ }^{3}$ Endocrinology Unit, Centre for Cardiovascular Science, University of Edinburgh, Queen's Medical Research Institute, Edinburgh, UK

\section{Correspondence to} Dr Sohinee Bhattacharya, Dugald Baird Centre for Research on Women's Health, Aberdeen Maternity Hospital, Aberdeen AB25 2ZL, UK; sohinee.bhattacharya@abdn. ac.uk

Received 18 September 2015 Revised 21 March 2016 Accepted 12 April 2016 Published Online First 12 May 2016

\section{CrossMark}

$$
\begin{aligned}
& \text { To cite: Bhattacharya S, } \\
& \text { McNeill G, Raja EA, et al. } \\
& \text { Heart 2016;102:1456- }
\end{aligned}
$$$$
1463 .
$$

\begin{abstract}
Objective To examine the effect of maternal gestational weight gain (GWG) on adult offspring mortality, cardiovascular morbidity and cerebrovascular morbidity.

Methods The Aberdeen Children of the Nineteen Fifties (ACONF) is a population-based cohort of adults born in Aberdeen, Scotland between 1950 and 1956. GWG of the mothers of cohort members was extracted from original birth records and linked to the data on offspring morbidity and mortality up to 2011 obtained from Scottish national records. HRs for cardiovascular events and mortality in offspring according to maternal weight gain in pregnancy were estimated adjusting for maternal and offspring confounders using a restricted cubic spline model.
\end{abstract}

Results After exclusions, 3781 members of the original ACONF cohort were analysed. Of these, 103 (2.7\%) had died, $169(4.5 \%)$ had suffered at least one cardiovascular event and 73 (1.9\%) had had a hospital admission for cerebrovascular disease. Maternal weight gain of $1 \mathrm{~kg} /$ week or more was associated with increased risk of cerebrovascular event in the offspring (adjusted HR 2.70 (95\% Cl 1.19 to 6.12)). There was no association seen between GWG and offspring's all-cause mortality or cardiovascular event. Adult offspring characteristics (smoking, body mass index (BMI) and diabetes) were strongly associated with each outcome.

Conclusions Maternal GWG above $0.9 \mathrm{~kg} /$ week may increase the risk of cerebrovascular disease in the adult offspring, but not all-cause mortality or cardiovascular disease. Health and lifestyle factors such as smoking, $\mathrm{BMI}$ and diabetes in the adult offspring had a stronger influence than maternal and birth characteristics on their mortality and morbidity.

\section{INTRODUCTION}

Excessive weight has established health risks for both the mother and baby during pregnancy, ${ }^{1}$ and in the longer term, including premature mortality. ${ }^{2}$ Proposed mechanisms for this long-term risk include genetic predisposition, shared environment and fetal programming of adult disease. ${ }^{4}$

The effect of maternal weight gain during pregnancy (gestational weight gain or GWG) on adult offspring health is less clear. Many of the cohorts designed to study the effects of maternal nutrition in pregnancy on offspring health are currently relatively young and therefore can only report adverse outcomes at the time of birth, childhood or young adulthood. Most of these have focused on offspring body mass index (BMI), with high correlations found with maternal GWG. Morrison et $a l^{5}$ found that maternal GWG was positively associated with insulin levels and birth weight, length and body fat in the newborn. The Jerusalem Perinatal Family Follow-up Study found that the offspring of mothers within the upper prepregnancy BMI quartile $\left(\mathrm{BMI}>26.4 \mathrm{~kg} / \mathrm{m}^{2}\right)$ had a higher BMI and cardiometabolic traits compared with those born to mothers in the lower quartile $\left(\mathrm{BMI}<21.0 \mathrm{~kg} / \mathrm{m}^{2}\right)$ at 32 years of age. ${ }^{6}$ These associations were independent of maternal GWG and other confounders.

Record linkage of a mature cohort-Aberdeen Children of the Nineteen Fifties (ACONF) to local obstetric and national vital statistics and hospital clinical datasets available in Scotland enabled us to test the hypothesis that maternal GWG is associated with subsequent cardiovascular morbidity and premature mortality in the adult offspring, independent of any effects of high maternal BMI, early in pregnancy and offspring characteristics.

\section{METHODS}

\section{Ethical approval}

Ethical approval for the ACONF study was obtained from the North of Scotland Research Ethics Service. Approval to access and link relevant data for this analysis was obtained from the Aberdeen Maternity and Neonatal Databank (AMND) Steering Committee, the steering committee of the ACONF study and the Privacy Advisory Committee of the NHS National Services Scotland.

\section{Data sources}

Data were obtained from four sources:

1. The ACONF study contains data on children born between 1950 and 1956 who attended school in Aberdeen city ${ }^{7}$ and formed the basis of the current investigation. The ACONF database contains sociodemographic variables about the children, as well as their height and weight measurements taken between 1962 and 1964 as part of a school survey. Information about adult height, weight, socioeconomic status and selfreported history of diabetes was obtained from a questionnaire follow-up of the cohort conducted in 2001.

2. The AMND is an obstetric database that records all pregnancy-related events occurring in Aberdeen since 1950 (http://www.abdn.ac.uk/ amnd). From this database, we obtained pregnancy and delivery details of the mothers of 
children in ACONF, including their age at delivery, height and antenatal weights recorded during each antenatal clinic visit.

3. The Scottish Morbidity Records (SMR) database contains details of all hospital admissions and discharges in Scotland since 1981 with the discharge diagnosis coded using International Classification of Diseases V.9 (ICD-9) to April 1996 and V.10 (ICD-10) thereafter.

4. The General Register Office provided date and cause of death information for the ACONF cohort.

\section{Record linkage}

The Community Health Index (CHI) number, a unique identifier attributed to all individuals registered with a general practice in Scotland was used for deterministic linkage. In addition, probabilistic matching, using surname, date of birth, gender and post code of residence, was used in cases where CHI number was missing. All linkages were carried out by the Data Management Team of the University of Aberdeen and the Information and Services Division of NHS Scotland. After linkage, identifying variables were removed to generate a pseudonymised dataset before transfer to the researchers for analysis.

\section{Data cleaning and exclusions}

We excluded ACONF members who did not complete the questionnaire survey in 2001, emigrated out of Scotland or did not report one or more of the adult characteristics. We also excluded all participants whose mothers did not have more than one weight recorded in pregnancy, or who had only two weights recorded less than 2 weeks apart (figure 1).

\section{Study design}

This was a cohort study in which the exposure was maternal GWG obtained by subtracting the first from the last recorded antenatal weight and dividing the difference by the number of weeks elapsed between the two recordings.

We considered three outcomes in the offspring: (i) all-cause mortality, (ii) any cardiovascular disease-mainly identified by a hospital admission due to cardiovascular disease ((ICD 10 codes I20-I25), arterial disease (I73-I74) or other cardiovascular disease)-as recorded in the SMR database or death from cardiovascular disease without any previous hospital admission for this condition and (iii) any hospital admission or death for cerebrovascular disease (ICD 10 codes I60-I69).

Covariates were adjusted for in a stepwise manner. Maternal level variables (age at delivery, maternal early pregnancy BMI

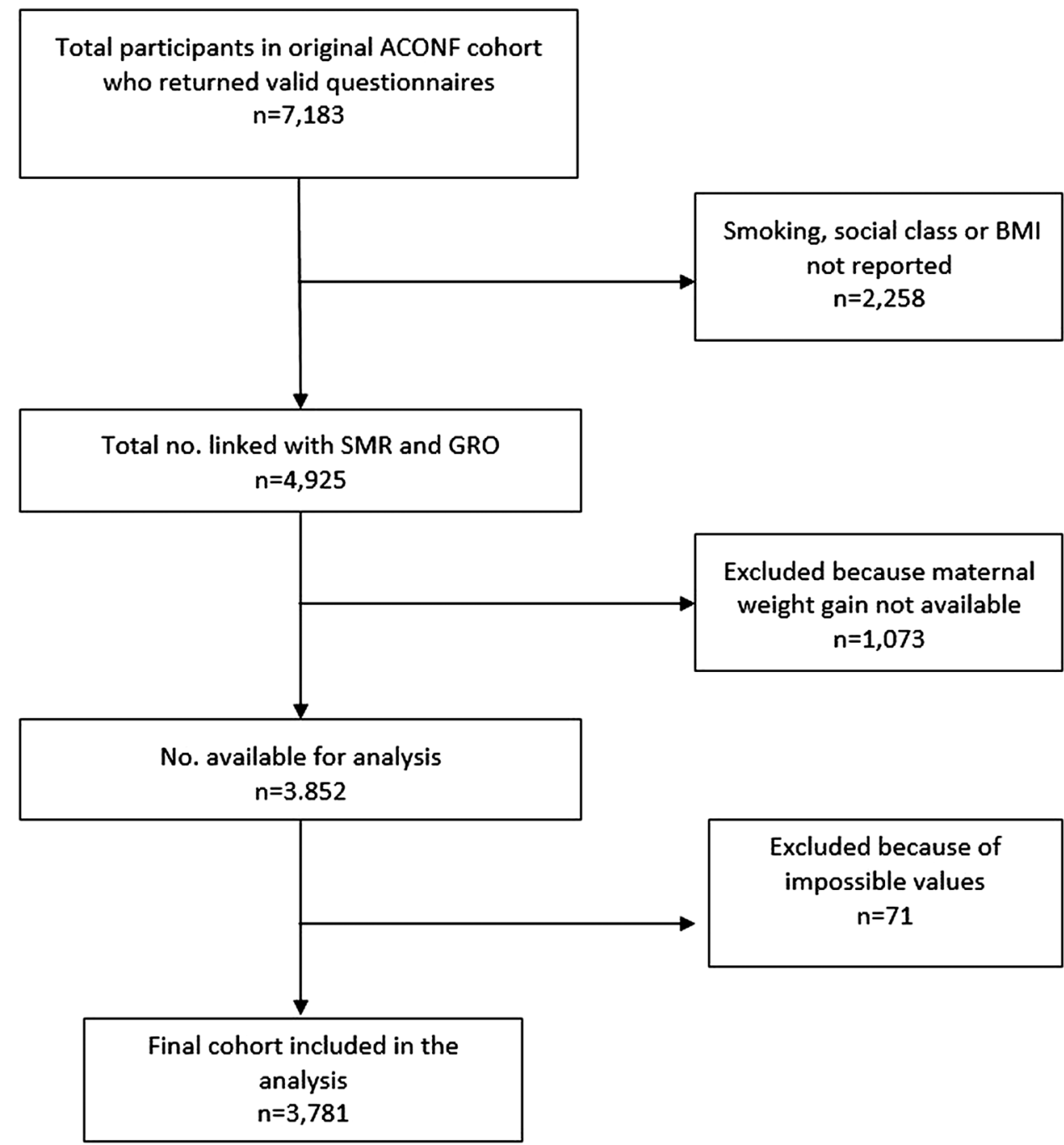

Figure 1 Flow chart of cohort follow-up with exclusions. ACONF, Aberdeen Children of the Nineteen Fifties; BMI, body mass index; SMR, Scottish morbidity records. GRO; General Registrar's Office. 
calculated from the height and weight recorded at the first antenatal clinic visit and social class according to the Registrar General's Classification of Occupations based on the social class of the father) were included in the adjusted model (model 2). In model 3, offspring level variables at the time of birth and childhood (gender, standardised birth weight score) ${ }^{8}$ childhood BMI SD Score (SDS) or z-score calculated using the LMS (Lambda$\mathrm{Mu}-$ Sigma) method ${ }^{9}$ from the height and weight recorded as part of the ACONF Reading Survey were included in addition to the covariates in model 2. Offspring's adult social class (based on the participant's employment socioeconomic group), ${ }^{10}$ adult smoking habits, adult BMI and self-reported history of diabetes mellitus, information collected as part of the ACONF follow-up survey in 2001 when participants were aged between 43 and 49 years of age, were included in the fourth and final model in addition.

The underlying time variable for the analysis was the age of the offspring at death, date of hospital discharge for the

Table 1 Comparison of maternal and offspring risk factors for offspring mortality, hospital admissions for cardiovascular and cerebrovascular disease

\begin{tabular}{|c|c|c|c|c|c|c|c|c|c|}
\hline Characteristics & $\begin{array}{l}\text { No death } \\
(n=3678)\end{array}$ & $\begin{array}{l}\text { Death } \\
(n=103)\end{array}$ & $p$ Value & $\begin{array}{l}\text { No CVD } \\
(n=3612)\end{array}$ & $\begin{array}{l}\text { Any CVD } \\
(n=169)\end{array}$ & p Value & $\begin{array}{l}\text { No cerebrovascular } \\
(\mathrm{n}=3708)\end{array}$ & $\begin{array}{l}\text { Cerebrovascular } \\
(n=73)\end{array}$ & $\mathrm{p}$ Value \\
\hline \multicolumn{10}{|l|}{ Maternal characteristics } \\
\hline GWG rate (kg/week)* & $0.41(0.16)$ & $0.40(0.16)$ & 0.53 & $0.41(0.16)$ & $0.41(0.17)$ & 0.97 & $0.41(0.16)$ & $0.43(0.21)$ & 0.40 \\
\hline \multicolumn{10}{|l|}{ Rate of GWG } \\
\hline$<0.2 \mathrm{~kg} /$ week & $266(7.2)$ & $9(8.7)$ & 0.31 & $258(7.1)$ & $17(10.1)$ & 0.82 & $271(7.3)$ & $4(5.5)$ & 0.88 \\
\hline $0.2-0.39 \mathrm{~kg} /$ week & $1490(40.5)$ & $44(42.7)$ & & $1466(40.6)$ & $68(40.2)$ & & $1498(40.4)$ & $36(49.3)$ & \\
\hline $0.4-0.59 \mathrm{~kg} /$ week & $1505(40.9)$ & $42(40.8)$ & & $1487(41.2)$ & $60(35.5)$ & & $1523(41.1)$ & $24(32.9)$ & \\
\hline $0.6-0.79 \mathrm{~kg} /$ week & $362(9.8)$ & $6(5.8)$ & & $348(9.6)$ & $20(11.8)$ & & $362(9.8)$ & $6(8.2)$ & \\
\hline$\geq 0.8 \mathrm{~kg} /$ week & $55(1.5)$ & $2(1.9)$ & & $53(1.5)$ & $4(2.4)$ & & $54(1.5)$ & $3(4.1)$ & \\
\hline $\begin{array}{l}\text { Age at delivery }{ }^{*} \text { (years) } \\
\text { Missing } n=10\end{array}$ & $27.27(5.20)$ & $27.61(5.63)$ & 0.51 & $27.31(5.22)$ & 26.59 (4.95) & 0.08 & $27.28(5.21)$ & $27.08(5.02)$ & 0.75 \\
\hline Maternal BMI ${ }^{*} \mathrm{~kg} / \mathrm{m}^{2}$ & $22.85(3.12)$ & $23.64(3.64)$ & 0.01 & $22.86(3.11)$ & $23.11(3.59)$ & 0.31 & $22.86(3.13)$ & $23.66(3.32)$ & 0.03 \\
\hline \multicolumn{10}{|l|}{ Maternal social class } \\
\hline I-IIla non-manual & $767(20.9)$ & $20(19.4)$ & 0.84 & $760(21.0)$ & $27(16.0)$ & 0.23 & $774(20.9)$ & $13(17.8)$ & 0.56 \\
\hline IIIb-V manual & $2509(68.2)$ & $73(70.9)$ & & $2457(68.0)$ & $125(74.0)$ & & $2528(68.2)$ & $54(74.0)$ & \\
\hline Missing & $402(10.9)$ & $10(9.7)$ & & $395(10.9)$ & $17(10.1)$ & & $406(11.0)$ & $6(8.2)$ & \\
\hline \multicolumn{10}{|c|}{ Offspring childhood characteristics } \\
\hline \multicolumn{10}{|l|}{ Offspring gender } \\
\hline Male & $1752(47.6) 1926$ & 55 (53.4) 48 & 0.25 & $1698(47.0)$ & $109(64.5)$ & $<0.01$ & $1764(47.6)$ & $43(58.9)$ & 0.06 \\
\hline Female & $(52.4)$ & $(46.6)$ & & $1914(53.0)$ & $60(35.5)$ & & $1944(52.4)$ & $30(41.1)$ & \\
\hline Offspring birth weight $(\mathrm{g})^{*}$ & $3323.13(477.48)$ & $\begin{array}{l}3377.12 \\
(516.50)\end{array}$ & 0.26 & $\begin{array}{l}3325.46 \\
(475.49)\end{array}$ & $\begin{array}{l}3306.49 \\
(542.10)\end{array}$ & 0.62 & 3323.78 (477.77) & $3366.81(520.65)$ & 0.45 \\
\hline $\begin{array}{l}\text { Offspring SBS* } \\
\text { Missing } n=372\end{array}$ & $0.01(0.97)$ & $0.14(0.99)$ & 0.20 & $0.02(0.97)$ & $-0.02(1.01)$ & 0.65 & $0.02(0.97)$ & $0.01(0.97)$ & 0.93 \\
\hline $\begin{array}{l}\text { Offspring BMI SDS* } \\
\text { Missing } n=112\end{array}$ & $0.47(0.88)$ & $0.67(0.84)$ & 0.03 & $0.48(0.88)$ & $0.55(0.90)$ & 0.28 & $0.48(0.88)$ & $0.60(0.92)$ & 0.26 \\
\hline \multicolumn{10}{|l|}{ Offspring adult characteristics } \\
\hline \multicolumn{10}{|l|}{ Offspring social class } \\
\hline SEG 1.1 to 4 & $1029(28.0)$ & $23(22.3)$ & 0.03 & $1012(28.0)$ & $40(23.7)$ & $<0.001$ & $1040(28.1)$ & $12(16.4)$ & 0.88 \\
\hline SEG 5.1 to 6 & $1542(41.9)$ & $40(38.8)$ & & $1525(42.2)$ & $57(33.7)$ & & $1559(42.0)$ & $23(31.5)$ & \\
\hline SEG 7 to 8 & $325(8.8)$ & $9(8.7)$ & & $319(8.8)$ & $15(8.9)$ & & $321(8.7)$ & $13(17.8)$ & \\
\hline SEG 9 to 16 & $782(21.3)$ & $31(30.1)$ & & $756(20.9)$ & $57(33.7)$ & & $788(21.3)$ & $25(34.3)$ & \\
\hline \multicolumn{10}{|l|}{ Offspring smoking } \\
\hline Current & $894(24.3)$ & $56(54.4)$ & $<0.001$ & $870(24.1)$ & $80(47.3)$ & $<0.01$ & $908(24.5)$ & $42(57.5)$ & $<0.01$ \\
\hline Ex-smoker & $943(25.6)$ & $21(20.4)$ & & $923(25.6)$ & $41(24.3)$ & & $945(25.5)$ & $19(26.0)$ & \\
\hline No & $1841(50.1)$ & $26(25.2)$ & & $1819(50.4)$ & $48(28.4)$ & & $1855(50.0)$ & $12(16.4)$ & \\
\hline \multicolumn{10}{|l|}{ Offspring adult BMI } \\
\hline Underweight & $21(0.6)$ & $4(3.9)$ & 0.17 & $23(0.6)$ & $2(1.2)$ & $<0.01$ & $25(0.7)$ & $0(0)$ & 0.88 \\
\hline Normal & $1555(42.3)$ & $51(49.5)$ & & $1560(43.2)$ & $46(27.2)$ & & $1567(42.3)$ & $39(53.4)$ & \\
\hline Overweight & 1446 (39.3) & $26(25.2)$ & & $1401(38.8)$ & $71(42.0)$ & & 1449 (39.1) & $23(31.5)$ & \\
\hline Obese & $656(17.8)$ & $22(21.4)$ & & $628(17.4)$ & $50(29.6)$ & & $667(18.0)$ & $11(15.1)$ & \\
\hline \multicolumn{10}{|l|}{ Diabetes } \\
\hline Yes & $61(1.7)$ & $5(4.9)$ & 0.02 & $53(1.5)$ & $13(7.7)$ & $<0.01$ & $60(1.6)$ & $6(8.2)$ & $<0.01$ \\
\hline No & 3617 (98.3) & $98(95.2)$ & & 3559 (98.5) & $156(92.3)$ & & $3648(98.4)$ & 67 (91.8) & \\
\hline
\end{tabular}

Statistically significant $\mathrm{p}$ values are shown as bold.

Presented as number (\%) unless otherwise stated.

CVD, cardiovascular disease; GWG, gestational weight gain; SBS, standardised birth weight score; SDS, SD score; SEG, socioeconomic group.

${ }^{*}$ Mean (SD). 
outcomes of interest or end of follow-up (31 January 2012), whichever occurred earliest.

\section{Statistical analysis}

Data were analysed using Stata/MP 13 (StataCorp, Texas, USA). Descriptive univariate analyses of the data were done initially. Cox's proportional hazards model was used to assess the relationship between maternal GWG and the prespecified health outcomes in their adult offspring. To allow for some children having siblings in the dataset, we adjusted for clustering on the mother using multilevel modelling. We estimated robust standard errors after adjusting for multiple offspring clustered within mothers. ${ }^{11}$

Rate of GWG was treated as a continuous variable in order not to lose information and to model any non-linear relationships. Unadjusted HRs and 95\% CI for the prespecified outcomes by the rate of weight gain ( $\mathrm{kg} /$ week) were calculated (model 1), followed by three adjusted models as described above. The proportional hazard assumption was tested using Schoenfeld residuals, ${ }^{12}$ and no violations were detected. To model the non-linear relationship between rate of weight gain and offspring outcomes, a restricted cubic spline (RCS) procedure was adopted. ${ }^{13}{ }^{14}$ This uses multiple polynomial line segments within the range of rate of weight gain, the boundaries of which are called knots. Knots were placed at equally spaced centiles of the distribution of rate of weight gain. In our analyses, five knots were considered, placed at the 0th, 25th, 50th, 75th and 100th percentiles; corresponding rates of weight gain values were $0.01,0.32,0.41,0.50$ and $1.35 \mathrm{~kg} /$ week, respectively. The spline function was assumed to be significant if the $\mathrm{p}$ value for the model $\chi^{2}$ was less than $5 \%$, and the association was assumed to be non-linear if the spline coefficients differed significantly from each other on the Wald test for linearity. A rate of weight gain of $0.4 \mathrm{~kg} /$ week was used as the reference value in these RCS Cox analyses as this corresponded to the 50th centile.

\section{Missing values}

Complete case analysis was done for missing data on exposure variables. Where data were missing in categorical covariates, a separate category was created for missing observations in each of the covariates and included in the relevant analyses.

Missing in continuous variables was treated as missing in the analysis.

In the modelling diagnostic, any outliers and influential data points were checked using likelihood displacement values and largest eigen values of the Matrix (LMAX) values ${ }^{15}$ for the final model. A scatter plot between predicted likelihood displacement values and time to event for each of the outcomes was used to identify any observations with disproportionate influence. Similarly, predicted LMAX was used instead of likelihood displacement measure. Four observations appeared to be somewhat influential relative to others. Those four observations were excluded, and the analyses were repeated for all the outcomes in the final model. The estimates of the covariates were almost same as the estimates with the observations included in the modelling.

Multiple imputation was carried out using REALCOM Impute, a software for multilevel multiple imputation. The multilevel multiple imputations were carried out for variables with missing observations using complete information on other covariates for all cases and outcomes. The results were compared between complete case analysis and complete+imputed dataset.

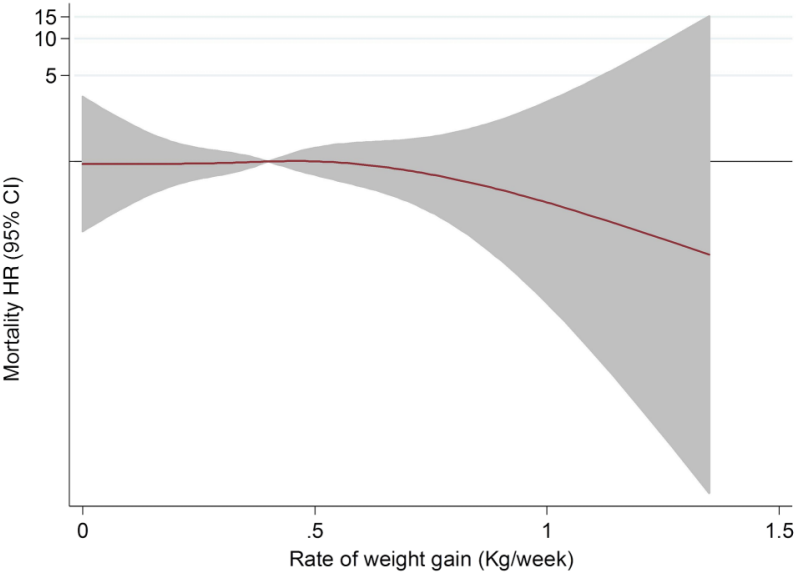

Figure 2 Fully adjusted HRs with $95 \%$ Cls for offspring mortality by maternal gestational weight gain.

\section{RESULTS}

Figure 1 shows cohort follow-up with exclusions. After applying all of the exclusion criteria described above, there were 3781 members of the original ACONF cohort $(n=12151)$ included in the analysis. Of these, $103(2.7 \%)$ had died, $169(4.5 \%)$ had suffered at least one cardiovascular event and 73 (1.9\%) had had a hospital admission or death from cerebrovascular disease. The major causes of death were neoplasms $(31.5 \%)$, diseases of the circulatory system (26.0\%), diseases of the digestive system $(10.0 \%)$, metabolic diseases $(8.3 \%)$ and injury or trauma $(6.2 \%)$.

Table 1 compares the baseline characteristics of those who did and did not experience the outcomes of all-cause mortality, or cardiac or cerebrovascular event. Members of the ACONF cohort who had died were more likely to have mothers with a higher BMI during pregnancy (mean 23.64 (SD 3.64) vs 22.85 (SD 3.12), $\mathrm{p}=0.01$ ); higher $\mathrm{BMI}$ in childhood expressed as SDS (mean $0.67(0.84)$ vs $0.47(0.88), p=0.03)$. As adults, they were more likely to belong to a more deprived socioeconomic status group ( $\mathrm{p}$ for trend $=0.03$ ), to be current smokers $(54.4 \%$ vs $24.3 \%, \mathrm{p}<0.01)$ and suffer from diabetes $(4.9 \%$ vs $1.7 \%$, $\mathrm{p}<0.01)$.

Compared with those who did not have a cardiovascular event, those who did were more likely to be male $(64.5 \%$ vs $47.0 \%, \mathrm{p}<0.01)$, and as adults belong to a more deprived

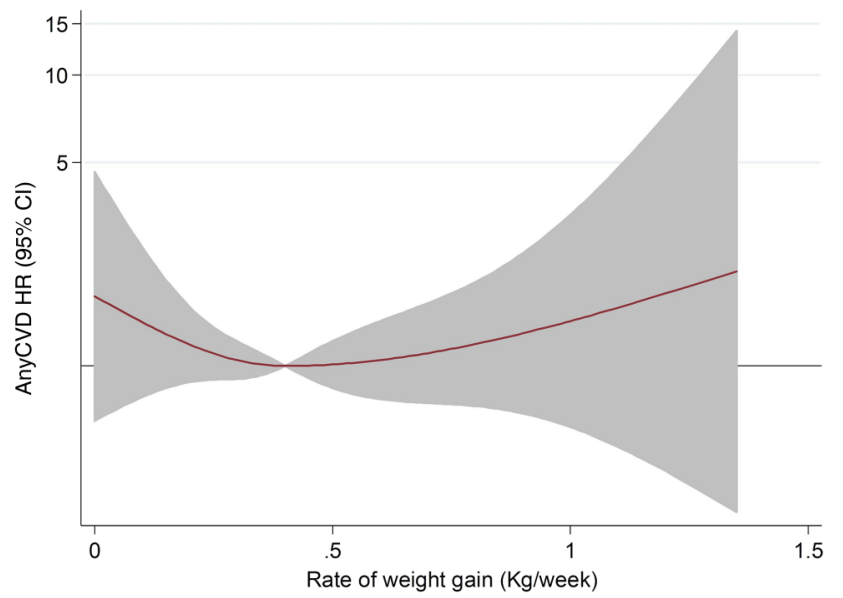

Figure 3 Adjusted HRs with $95 \%$ Cls of any cardiovascular disease event in the offspring by rate of maternal gestational weight gain. CVD, cardiovascular disease. 


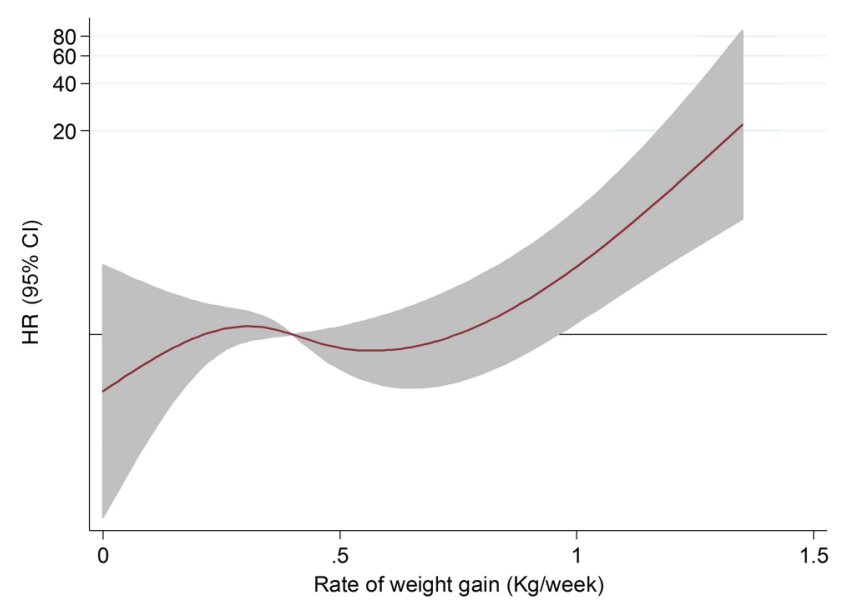

Figure 4 Adjusted HRs with $95 \%$ Cls for mortality due to or any cerebrovascular disease event in the offspring by maternal gestational weight gain.

socioeconomic status group ( $\mathrm{p}$ for trend $<0.01$ ), currently smoke $(47.3 \%$ vs $24.1 \%, \mathrm{p}<0.01$ ), have a higher BMI ( $\mathrm{p}$ for trend $<0.01)$ and report diabetes $(7.7 \%$ vs $1.5 \%, \mathrm{p}<0.01)$.

Those who had had a cerebrovascular event were more likely to have mothers with a higher BMI in pregnancy (mean 23.66 (SD 3.32$) \mathrm{kg} / \mathrm{m}^{2}$ vs 22.86 (SD 3.13 ) $\mathrm{kg} / \mathrm{m}^{2}, \mathrm{p}=0.03$ ). As adults, they were more likely to be current smokers $(57.5 \%$ vs $24.5 \%$, $\mathrm{p}<0.01)$ and diabetic $(8.2 \%$ vs $1.6 \%, \mathrm{p}<0.01)$.

Of note, rate of maternal GWG was not associated with any of the outcomes of interest in the offspring on univariate analysis.

Figures 2-4 show respectively the relationship between maternal GWG and the offspring's risk of all-cause mortality, hospital admission for any cardiovascular disease and hospital admission for any cerebrovascular condition, from the fully adjusted model. The HRs with 95\% CIs for these outcomes at each node of GWG are presented in table 2, with results from each model shown separately in a stepwise fashion.

Association between GWG and offspring all-cause mortality

Neither the unadjusted nor any of the adjusted models showed a statistically significant association between maternal GWG and offspring risk of all-cause mortality (table 2). Figure 2 is the visual representation of the fully adjusted HRs with 95\% CIs for offspring mortality by maternal GWG. According to this figure, there appears to be a reduction in offspring mortality risk with increased GWG, although the association was not statistically significant.

\section{Association between GWG and offspring cardiovascular event}

The adjusted and unadjusted HRs with 95\% CIs of any hospital admission or death from cardiovascular disease in the offspring by maternal GWG are presented in table 2. Cardiovascular disease in the offspring did not show statistically significant association with maternal GWG in any of the unadjusted or adjusted models. Figure 3 demonstrates the relationship between maternal GWG and hospital admission for any cardiovascular event in the offspring adjusted for confounding factors. Although not statistically significant, this figure shows a U-shaped relationship with higher risk of cardiovascular events at both extremes of maternal GWG.

\section{Association between GWG and offspring cerebrovascular event}

Table 2 and figure 4 present the relationship between maternal GWG and offspring risk of any cerebrovascular event. As table 2

Table 2 Cox proportional hazards models for association between rate of GWG (kg/week) and offspring mortality, cardiovascular or cerebrovascular disease through restricted cubic splines

\begin{tabular}{|c|c|c|c|c|}
\hline \multirow[b]{2}{*}{ Rate of GWG } & \multicolumn{3}{|l|}{$\mathrm{HR}(95 \% \mathrm{Cl})$} & \multirow[b]{2}{*}{ Model $4(n=3296)$} \\
\hline & Model 1 (n=3781) & Model 2 (n=3771) & Model 3 (n=3296) & \\
\hline \multicolumn{5}{|c|}{ Offspring mortality } \\
\hline $0.2 \mathrm{~kg} /$ week & $1.13(0.80$ to 1.60$)$ & $1.02(0.72$ to 1.47$)$ & $1.01(0.70$ to 1.48$)$ & $0.94(0.64$ to 1.40$)$ \\
\hline 0.4 kg/week & 1.00 & 1.00 & 1.00 & 1.00 \\
\hline $0.6 \mathrm{~kg} /$ week & $1.02(0.70$ to 1.48$)$ & 1.01 (0.70 to 1.46$)$ & $0.95(0.64$ to 1.41$)$ & $0.96(0.6341 .43)$ \\
\hline $0.8 \mathrm{~kg} /$ week & $0.86(0.35$ to 2.11$)$ & $0.82(0.33$ to 2.01$)$ & 0.77 (0.32 to 1.82$)$ & $0.73(0.31$ to 1.73$)$ \\
\hline $1.0 \mathrm{~kg} /$ week & $0.63(0.08$ to 5.03$)$ & 0.57 (0.07 to 4.48$)$ & 0.55 (0.08 to 3.74$)$ & $0.47(0.07$ to 3.10$)$ \\
\hline \multicolumn{5}{|c|}{ Any cardiovascular disease (CVD) in offspring } \\
\hline $0.2 \mathrm{~kg} /$ week & $1.17(0.91$ to 1.51$)$ & 1.16 (0.87 to 1.53$)$ & 1.20 (0.90 to 1.60$)$ & $1.20(0.89$ to 1.61$)$ \\
\hline $0.4 \mathrm{~kg} /$ week & 1.00 & 1.00 & 1.00 & 1.00 \\
\hline $0.6 \mathrm{~kg} /$ week & $1.08(0.81$ to 1.44$)$ & $1.05(0.79$ to 1.40$)$ & 1.06 (0.78 to 1.43$)$ & $1.03(0.76$ to 1.40$)$ \\
\hline $0.8 \mathrm{~kg} /$ week & 1.31 (0.83 to 2.07$)$ & 1.23 (0.78 to 1.94$)$ & $1.21(0.75$ to 1.97$)$ & $1.16(0.71$ to 1.88$)$ \\
\hline $1.0 \mathrm{~kg} /$ week & 1.74 (0.80 to 3.76$)$ & 1.54 (0.70 to 3.39$)$ & 1.50 (0.66 to 3.42$)$ & 1.37 (0.59 to 3.18$)$ \\
\hline \multicolumn{5}{|c|}{ Any cerebrovascular disease in offspring } \\
\hline $0.2 \mathrm{~kg} /$ week & 1.07 (0.66 to 1.74$)$ & $0.98(0.60$ to 1.60$)$ & 1.10 (0.67 to 1.79$)$ & $0.97(0.59$ to 1.60$)$ \\
\hline $0.4 \mathrm{~kg} /$ week & 1.00 & 1.00 & 1.00 & 1.00 \\
\hline $0.6 \mathrm{~kg} /$ week & $0.78(0.49$ to 1.25$)$ & 0.74 (0.46 to 1.19$)$ & 0.81 (0.48 to 1.35$)$ & $0.80(0.47$ to 1.34$)$ \\
\hline $0.8 \mathrm{~kg} /$ week & 1.21 (0.64 to 2.29$)$ & 1.11 (0.59 to 2.10$)$ & 1.30 (0.65 to 2.61$)$ & $1.16(0.57$ to 2.40$)$ \\
\hline $1.0 \mathrm{~kg} /$ week & 3.19 (1.43 to 7.09$)$ & 2.83 (1.31 to 6.12$)$ & 3.55 (1.60 to 7.92 ) & 2.70 (1.19 to 6.12 ) \\
\hline \multicolumn{5}{|c|}{$\begin{array}{l}\text { Model 1: rate of GWG as continuous variable in non-linear form (cubic spline). } \\
\text { Model 2: model 1+maternal factors—-age at delivery, BMI and social class. } \\
\text { Model 3: model 2+offspring factors—gender, SBS, childhood BMI SDS. } \\
\text { Model 4: model 3+smoking, adult social class, adult BMI and diabetes. } \\
\text { Statistically significant HRs are shown as bold. } \\
\text { GWG, gestational weight gain; SBS, standardised birth weight score; SDS, SD score. }\end{array}$} \\
\hline
\end{tabular}


shows, a weight gain of $1 \mathrm{~kg} /$ week or more was associated with an increased risk of cerebrovascular event in the offspring in the unadjusted model (HR 3.19 (95\% CI 1.43 to 7.09)), the model adjusted for maternal factors only (adj. HR 2.83 (95\% CI 1.31 to 6.12)), the model adjusted for maternal and offspring's birth and childhood level factors (adj. HR $3.55 \quad$ (95\% CI 1.60 to 7.92)) and the fully adjusted model additionally adjusting for adult offspring-level factors (adj.HR $2.70 \quad$ (95\% $\quad$ CI 1.19 to 6.12$)$ ).

Table 3 presents the HRs with 95\% CIs for each of the variables included in the fully adjusted models, which show that the characteristics of offspring as adults are the main drivers of risk of all-cause mortality, cardiovascular disease and cerebrovascular disease. Being a current smoker when surveyed in 2001 was strongly associated with mortality (adj HR 4.10 (95\% CI 2.50 to 6.74)), cardiovascular disease (adj HR 3.32 (95\% CI 2.29 to 4.81)) and cerebrovascular disease (adj HR 5.45 (95\% CI 2.71 to 10.93$)$ ). Being diabetic also carried a higher risk of all-cause mortality (adj HR 2.79 (95\% CI 1.09 to 7.11)), cardiovascular disease (adj HR 4.05 (95\% CI 2.23 to 7.33)) and cerebrovascular disease (adj HR 6.41(95\% CI 2.85 to 14.42)). Adult offspring BMI showed inconsistent associations with the outcomes of interest-while being underweight was associated with mortality (adj HR 4.16 (95\% CI 1.28 to 13.49)), overweight (adj HR 1.63 (95\% CI 1.11 to 2.39)) and obesity (adj HR 2.65 (95\% CI 1.71 to 4.11$)$ ) were associated with increased risk of cardiovascular disease but not cerebrovascular disease.

In the secondary analysis using dataset with multiple imputations, the results were comparable with the analysis with complete cases. Only for the outcome of cerebrovascular disease in the offspring, widening CIs of effect estimates with increasing GWG meant there was no longer a statistically significant association seen.

\section{DISCUSSION}

We did not find a statistically significant relationship between maternal GWG and offspring all-cause mortality or cardiovascular events. Being overweight or obese as adults conferred a higher risk of cardiovascular events, whereas higher maternal

Table 3 Factors associated with offspring mortality/CVD/cerebrovascular disease using Cox proportional hazards model (fully adjusted model: model 4)

\begin{tabular}{|c|c|c|c|}
\hline Characteristics & Death HR $(95 \% \mathrm{Cl})$ & Any CVD HR (95\% Cl) & Any cerebrovascular $\mathrm{HR}(95 \% \mathrm{Cl})$ \\
\hline \multicolumn{4}{|l|}{ Maternal characteristics } \\
\hline \multicolumn{4}{|l|}{ Rate of weight gain } \\
\hline $0.2 \mathrm{~kg} /$ week & 0.94 (0.64 to 1.40$)$ & 1.20 (0.89 to 1.61$)$ & 0.97 (0.59 to 1.60$)$ \\
\hline $0.6 \mathrm{~kg} /$ week & $0.96(0.64$ to 1.43$)$ & $1.03(0.76$ to 1.40$)$ & $0.80(0.47$ to 1.34$)$ \\
\hline $0.8 \mathrm{~kg} /$ week & $0.73(0.31$ to 1.73$)$ & 1.16 (0.71 to 1.88$)$ & $1.16(0.57$ to 2.40$)$ \\
\hline $1.0 \mathrm{~kg} /$ week & $0.47(0.07$ to 3.10$)$ & 1.37 (0.59 to 3.18$)$ & 2.70 (1.19 to 6.12$)$ \\
\hline Age at delivery & $1.01(0.97$ to 1.05$)$ & 0.98 (0.96 to 1.02$)$ & $1.00(0.95$ to 1.04$)$ \\
\hline Maternal BMI & 1.05 (0.99 to 1.13$)$ & $1.00(0.94$ to 1.06$)$ & $1.06(0.98$ to 1.15$)$ \\
\hline \multicolumn{4}{|l|}{ Maternal social class } \\
\hline I-IIla non-manual & 1 & 1 & 1 \\
\hline Illb-V manual & $1.07(0.62$ to 1.83$)$ & 1.15 (0.74 to 1.78$)$ & $0.92(0.48$ to 1.79$)$ \\
\hline Missing & 1.07 (0.47 to 2.42$)$ & $1.32(0.69$ to 2.50$)$ & $0.54(0.15$ to 2.00$)$ \\
\hline \multicolumn{4}{|c|}{ Infant/childhood characteristics } \\
\hline \multicolumn{4}{|l|}{ Offspring gender } \\
\hline Female & 1 & 1 & 1 \\
\hline Male & $1.26(0.81$ to 1.97$)$ & 1.89 (1.33 to 2.67$)$ & 1.81 (1.04 to 3.15$)$ \\
\hline Offspring SBS & 1.10 (0.88 to 1.38$)$ & 0.98 (0.82 to 1.16$)$ & $0.92(0.71$ to 1.19$)$ \\
\hline Childhood BMI SDS & $1.23(0.97$ to 1.55$)$ & 0.95 (0.80 to 1.11$)$ & 1.09 (0.83 to 1.44$)$ \\
\hline \multicolumn{4}{|c|}{ Offspring adult characteristics } \\
\hline \multicolumn{4}{|l|}{ Offspring social class } \\
\hline SEG 1.1 to 4 & 1 & 1 & 1 \\
\hline SEG 5.1 to 6 & 1.20 (0.67 to 2.17 & $1.24(0.81$ to 1.90$)$ & 2.02 (0.94 to 4.35$)$ \\
\hline SEG 7 to 8 & $1.19(0.52$ to 2.73$)$ & 1.03 (0.55 to 1.92$)$ & 3.14 (1.25 to 7.88$)$ \\
\hline SEG 9 to 16 & 1.27 (0.71 to 2.26$)$ & $1.46(0.95$ to 2.25$)$ & 2.23 (1.03 to 4.86$)$ \\
\hline \multicolumn{4}{|l|}{ Offspring smoking } \\
\hline No & 1 & 1 & 1 \\
\hline Current & $4.10(2.50$ to 6.74$)$ & 3.32 (2.29 to 4.81$)$ & $5.45(2.71$ to 10.93$)$ \\
\hline Ex-smoker & 1.64 (0.89 to 3.04$)$ & 1.38 (0.89 to 2.15$)$ & 2.37 (1.08 to 5.18$)$ \\
\hline \multicolumn{4}{|l|}{ Offspring adult BMI } \\
\hline Underweight & 4.16 (1.28 to 13.49$)$ & 3.07 (0.73 to 12.93$)$ & 0 \\
\hline Normal & 1 & 1 & 1 \\
\hline Overweight & $0.52(0.31$ to 0.87$)$ & $1.63(1.11$ to 2.39$)$ & 0.57 (0.32 to 1.03$)$ \\
\hline Obese & 0.85 (0.48 to 1.50$)$ & 2.65 (1.71 to 4.11$)$ & $0.57(0.25 .1 .33)$ \\
\hline \multicolumn{4}{|l|}{ Diabetes } \\
\hline No & 1 & 1 & 1 \\
\hline Yes & 2.79 (1.09 to 7.11$)$ & 4.05 (2.23 to 7.33 ) & 6.41 (2.85 to 14.42$)$ \\
\hline
\end{tabular}


BMI during pregnancy was associated with an increased risk of cerebrovascular but not cardiovascular events on univariate analysis.

A key strength of this study was the well-defined cohort with adequate length of follow-up to detect outcomes of interest. Even so the relatively small number of outcomes may have limited our power to detect associations that really exist, especially at the extremes of maternal GWG. Another strength of the study was the high-quality data used for the analysis. ${ }^{16}$ Linkage with ISD and GRO in Scotland by first deterministic (where possible) and then probabilistic matching maximised linkages and ensured a high proportion of true linkages. ${ }^{17}$ The availability of data at various time points during the life course of the offspring allowed the examination of risk factors at the time of delivery, offspring's childhood and middle-age adulthood. We were able to take account of clustering and collinearity within and between variables by using multilevel modelling. The cubic spline analysis enabled us to model the non-linear relationship between GWG and offspring morbidity and mortality without losing information through categorisation.

The main limitation of this study is the exclusion of a large proportion of the original cohort because of missing information on GWG (mostly due to a single weight being recorded during pregnancy), or non-response to the ACONF follow-up questionnaire. A comparison of cohort members with and without complete information showed that they differed in terms of gender, parents' marital status, social class at birth or in childhood but not in maternal GWG. ${ }^{18}$ As the SMR database was only initiated in 1981, left truncation of the outcome data would have occurred, although the oldest cohort members would have been 31 years old in 1981, an age when cardiovascular risk is low and mainly confined to congenital or rheumatic heart diseases. Fewer women were obese in pregnancy in the 1950 s, reducing generalisability of the findings to contemporary situations. As with all observational studies, residual confounding from unmeasured or poorly measured covariates may have affected our results.

It is difficult to tease out the effects of genetic predisposition, fetal programming and shared environment when studying the effects of maternal GWG on offspring morbidity and mortality later in life. Lawlor et $a l^{19}$ showed that neither maternal nor fetal adiposity-related genetic variants were associated with higher GWG. Nevertheless, higher GWG signifies higher birth weight which in turn is associated with higher risk of childhood and adult obesity. ${ }^{20-24}$

Far less is known about the impact of maternal GWG on offspring cardiovascular health. Some studies report a modest increase in blood pressure in children ${ }^{25}$ and young adults ${ }^{26-28}$ associated with high GWG. The synergistic mechanisms and the differences between maternal prepregnancy weight per se and GWG on the offspring's cardiovascular health warrant further discussion. GWG may be about nutritional content of the food consumed-particularly those gaining a lot of weight. The availability of adipose stores versus available fuel from food is likely to have differing effects on fetal growth and ultimately on future health in adulthood.

There is currently no agreement on whether mothers who are overweight or obese at the start of their pregnancy should limit their weight gain. In 2009, the US Institute of Medicine recommended that mothers with BMI in the range $25-30 \mathrm{~kg} / \mathrm{m}^{2}$ should gain $7-11.5 \mathrm{~kg}$ over the whole of pregnancy and $0.23-$ $0.33 \mathrm{~kg} /$ week in the second and third trimesters, with corresponding figures of $5-9 \mathrm{~kg}$ total gain and $0.17-0.27 \mathrm{~kg} /$ week in the second and third trimesters in those with a prepregnancy
BMI of 30 or more. ${ }^{29}$ In the UK, the National Institute for Health and Care Excellence concluded in 2010 that maternal weight should not be routinely monitored during pregnancy. ${ }^{30}$ Our findings are broadly reassuring, since maternal GWG per se was not associated with an increased risk of all-cause mortality and cardiovascular outcomes in the offspring. In comparison, risk factors measured in the offspring as adults had a stronger relationship with the outcomes. This indicates that being healthy as an adult (ie, being a non-smoker, having a healthy weight and being non-diabetic) is more important than any risks acquired in utero and in childhood. Longer-term follow-up of this cohort to accumulate cardiovascular events will allow subgroup analysis of mothers with high prepregnancy BMI to contribute to the debate on benefits of GWG restriction in overweight and obese women.

\section{CONCLUSION}

In this population-based cohort, GWG of $1 \mathrm{~kg} /$ week or more was associated with an increased risk of cerebrovascular disease in the adult offspring, an effect independent of maternal and offspring BMI as a child and adult. Maternal GWG was not associated with an increased risk of cardiovascular disease or allcause mortality in the adult offspring. Health and lifestyle factors in the adult offspring were the strongest determinants of their morbidity and mortality.

\section{Key messages}

What is already known on this subject?

Maternal prepregnancy body mass index and total gestational weight gain have been shown to affect cardiovascular parameters such as blood pressure in the young adult offspring. None of the published studies had adequate follow-up time to assess the effects on cardiovascular events and mortality.

\section{What might this study add?}

In a cohort follow-up study spanning 60 years, rate of gestational weight gain (GWG) was not found to be associated with offspring's risk of mortality or cardiovascular events. GWG of $0.9 \mathrm{~kg} /$ week or more was associated with increased risk of cerebrovascular events in the offspring. Adult health and lifestyle factors such as smoking, diabetes and obesity were strongly associated with the offspring's risk of mortality and morbidity.

\section{How might this impact on clinical practice?}

For the first time, this large-scale cohort study was able to show that adult health and lifestyle factors and not early life risk factors played the most important role in determining cardiovascular mortality and morbidity. Modifying these risk factors (obesity, smoking and diabetes) would constitute effective preventive strategy, irrespective of early life risk factors.

Acknowledgements The authors wish to thank and acknowledge the scientific input of Dr Nadeem Sarwar who led the funding application to carry out this research; Ms Katie Wilde and the Data Management Team, University of Aberdeen, for data extraction from the Aberdeen databases and Ms Lena Henderson from ISD Scotland for data linkage and extraction from SMR databases.

Contributors SB designed the study, facilitated data extraction and wrote the first draft of the manuscript; GMcN designed the study, supervised running of the project and commented on the analysis and draft manuscript; EAR conducted the statistical 
analyses; KA cleaned the data, conducted the initial analyses and commented on the draft manuscript; HC helped to extract and interpret the data from ACONF; PCH contributed to study design, study support, data interpretation and comments on manuscript; RMR and JEN helped to clinically interpret the findings and commented on the manuscript. SB is the guarantor for this paper.

Funding This research was supported by Chest Heart and Stroke Scotland (R10/ A128). The University of Aberdeen acted as sponsor for this research project, but the findings and the interpretation in this study are the authors' own.

Competing interests None declared.

Ethics approval Ethical approval for the Aberdeen Children of the 1950s study was obtained from the North of Scotland Research Ethics Service. Approval to access and link relevant data for this analysis was obtained from the Aberdeen Maternity and Neonatal Databank steering committee, the steering committee of the Aberdeen Children of the 1950s study and the Privacy Advisory Committee of the NHS National Services Scotland

Provenance and peer review Not commissioned; externally peer reviewed.

Data sharing statement Data for the Aberdeen Children of the Nineteen Fifties Cohort study can be obtained by applying to the steering group. Linked data from Information and Services Division of NHS Scotland can be obtained from eDRIS@nhs.net.

\section{REFERENCES}

1 Catalano PM, Ehrenberg HM. The short- and long-term implications of maternal obesity on the mother and her offspring. BJOG 2006;113:1126-33.

2 Drake AJ, Reynolds RM. Impact of maternal obesity on offspring obesity and cardiometabolic disease risk. Reproduction 2010;140:387-98.

3 Reynolds RM, Allan KM, Raja EA, et al. Maternal obesity during pregnancy and premature mortality from cardiovascular event in adult offspring: follow-up of 1323 275 person years. BMJ 2013;347:f4539.

4 Poston L. Maternal obesity, gestational weight gain and diet as determinants of offspring long term health. Best Pract Res Clin Endocrinol Metab 2012;26:627-39.

5 Morrison KM, Anand SS, Yusuf S, et al. Maternal and pregnancy related predictors of cardiometabolic traits in newborns. PLOS ONE 2013;8:e55815.

6 Hochner $\mathrm{H}$, Friedlander Y, Calderon-Margalit R, et al. Associations of maternal prepregnancy body mass index and gestational weight gain with adult offspring cardiometabolic risk factors: The Jerusalem perinatal family follow-up study. Circulation 2012;125:1381-9.

7 Leon DA, Lawlor DA, Clark $\mathrm{H}$, et al. Cohort profile: the Aberdeen children of the 1950s study. Int J Epidemiol 2006;35:549-52.

8 Campbell D, Hall M, Lemon J, et al. Clinical birthweight standards for a total population in the 1980s. Br J Obstet Gynaecol 1993;100:436-45.

9 Cole TJ. The LMS method for constructing normalized growth standards. Eur I Clin Nutr 1990:44:45-60.

10 Elias P. Social class and the standard occupational classifications. A Report on Phase 1 of the ESRC Review of the OPCS Social Classification. 1995.

11 Wei LJ, Lin DY, Weissfeld L. Regression analysis of multivariate incomplete failure time data by modelling marginal distributions. J Am Stat Assoc 1989;84:1065-73.
12 Hess KR. Graphical methods for assessing violations of the proportional hazards assumption in Cox regression. Stat Med 1995;14:1707-23.

13 Dupont W. Repeated-measures analysis of variance. Statistical Modeling for Biomedical Researchers: A Simple Introduction to the Analysis of Complex Data. 2002.

14 Greenland S. Dose-Response and trend analysis in epidemiology: alternatives to categorical analysis. Epidemiology 1995;6:356-65.

15 Collet D. Modelling survival data in medical research. 2nd edn. London: Chapman \& Hall/ CRC, 2003.

16 Atherton IM, Lynch E, Williams AJ, et al. Barriers and Solutions to Linking and Using Health and Social Care Data in Scotland. Br J Soc Work 2015;45:1614-22.

17 Fleming M, Kirby B, Penny KI. Record linkage in Scotland and its applications to health research. J Clin Nurs 2012;21:2711-21.

18 Nishiwaki Y, Clark H, Morton SM, et al. Early life factors, childhood cognition and postal questionnaire response rate in middle age: the Aberdeen children of the 1950s study. BMC Med Res Methodol 2005;5:16.

19 Lawlor DA, Fraser A, Macdonald-Wallis C, et al. Maternal and offspring adiposity-related genetic variants and gestational weight gain. Am J Clin Nutr 2011;94:149-55

20 Sewell MF, Huston-Presley L, Super DM, et al. Increased neonatal fat mass, not lean body mass, is associated with maternal obesity. Obstet Gynecol 2006;195: $1100-3$

21 Crozier SR, Inskip HM, Godfrey KM, et al. Weight gain in pregnancy and childhood body composition: findings from the Southampton Women's Survey. Am J Clin Nutr 2010;91:1745-51.

22 Nohr EA, Vaeth M, Baker JL, et al. Combined associations of prepregnancy body mass index and gestational weight gain with the outcome of pregnancy. Am J Clin Nutr 2008;87:1750-9.

23 Hull HR, Dinger MK, Knehans AW, et al. Impact of maternal body mass index on neonate birthweight and body composition. Obstet Gynecol 2008;198:416.e1-e6.

24 Viswanathan M, Siega-Riz AM, Moos MK, et al. Outcomes of Maternal Weight Gain. Rockville (MD): Agency for Healthcare Research and Quality (US); 2008 May. (Evidence Reports/Technology Assessments, No. 168.) Available from: http://www. ncbi.nlm.nih.gov/books/NBK38595/ (accessed 21 Aug 2015).

25 Oken E, Kleinman KP, Belfort MB, et al. Associations of gestational weight gain with short- and longer-term maternal and child health outcomes. Am J Epidemiol 2009;170:173-80.

26 Mamun AA, O'Callaghan M, Callaway L, et al. Associations of gestational weight gain with offspring body mass index and blood pressure at 21 years of ageevidence from a birth cohort study. Circulation 2009;119:1720-7.

27 Fraser A, Tilling K, MacDonald-Wallis $\mathrm{C}$, et al. Association of maternal weight gain in pregnancy with offspring obesity and metabolic and vascular traits in childhood. Circulation 2010;121:2557-64.

28 Smith GD, Steer C, Leary S, et al. Is there an intrauterine influence on obesity? Evidence from parent-child associations in the Avon Longitudinal Study of Parents and Children (ALSPAC). Arch Dis Child 2007;92:876-80.

29 Institute of Medicine and National Research Council. Weight Gain during Pregnancy: Reexamining the Guidelines. 2009.

30 Antenatal care: routine care for the healthy pregnant woman. NICE Clinical Guideline 62. 2010. 